\title{
Genetic Risks to the Mother and the Infant: Assessment, Counseling, and Management
}

\author{
Stuart K. Shapira $\cdot$ Siobhan Dolan
}

Published online: 20 June 2006

(C) Springer Science+Business Media, Inc. 2006

Key Words Prenatal genetic risks · Family history assessment · Advanced maternal age · Advanced paternal age $\cdot$ Preconception genetic counseling $\cdot$ Pregnancy risks from genetic conditions

In order to optimize the chance for a healthy pregnancy and baby, a woman's risk factors related to a variety of health issues (diet, medications, and exposures) are generally examined, allowing for required modifications during preconception care [1]. This activity can help prevent some birth defects and some adverse birth outcomes, such as prematurity. In contrast, genetic factors result in conditions often perceived to be rare, and thus most women without a family history of birth defects or genetic conditions do not consider themselves at risk. Regardless, a full range of genetic issues should always be considered preconceptionally to improve the chance for a healthy birth outcome.

Preconception care involves genetics in two distinct manners: 1) the assessment of risk factors for having a child with a genetic disorder and providing information about preconception and prenatal testing, as well a reproductive options to minimize the chance of having genetic problems in future

The findings and conclusions in this report are those of the author(s) and do not necessarily represent the views of the Centers for Disease Control and Prevention.

S. K. Shapira $(\square)$

NCBDDD, Centers for Disease Control and Prevention, 1600 Clifton Rd., NE, Mailstop E-86, Atlanta, GA, 30333 e-mail: cso6@cdc.gov

S. Dolan

Albert Einstein College of Medicine and March of Dimes, New York, NY children; and 2) the care of women with genetic disorders preconceptionally and during pregnancy.

Very few studies have evaluated the extent of genetic issues that are currently addressed in preconceptional care venues since genetic concerns often do not become important to a woman and/or her healthcare providers until she is pregnant. The most comprehensive examination of reproductive health occurs in the Hungarian periconceptual health care service, which has 20 years of experience in protecting the health of potential mothers through pregnancy, reducing the occurrence of unsuccessful pregnancy outcomes (including birth defects and genetic diseases), and promoting the birth of healthy infants $[2,3]$. Through this service, couples undergo comprehensive health screening, family history assessment, referral for carrier screening for cystic fibrosis, and predictive genetic testing based on family history, when appropriate. Unlike the Hungarian experience, there has not been a concerted approach toward genetic risk assessment during the preconception period in the United States because the first visit for medical care is usually an antenatal visit in the 6-12th week of gestation. Therefore, standards need to be developed for the incorporation of genetic risk assessments and counseling into a preconception care program.

Preconception genetic care should focus on providing an understanding of risk-specifically, assessing the risks and communicating appropriate information via non-directive counseling about birth defects and genetic disorders that may occur during a pregnancy. All couples have a baseline population risk of having an infant with a major birth defect or genetic problem. This empirical risk is $2-5 \%$, and is based on population data [4-6]. This conglomerate risk for any of the full spectrum of birth defects, chromosomal abnormalities, and single gene disorders does not take into account the individualized risks for a woman's future pregnancy, such as those based on familial factors, ethnic background, and 
parental age, all of which may increase the risk to one above the population risk. Given the reality of the general and the specific risks for each woman, a thorough individualized risk assessment and the provision of appropriate genetic counseling information are necessary for a couple to understand their genetic risks for a pregnancy.

A family history assessment, often performed by a genetic counselor, genetic nurse, or clinical geneticist, is used to determine familial factors that may increase the risk for genetic problems in a future pregnancy. Unfortunately, most women do not receive medical services from such healthcare providers unless there is a known or suspected genetic problem in the family. Therefore, self-assessment tools have been developed for obtaining a family history and alerting women who, based on increased risk factors determined by the assessment tool, should seek further genetic counseling [7-11]. Familial risk factors can include a family history of single gene disorders such as muscular dystrophy, cystic fibrosis, sickle cell disease, and Fragile X syndrome [12, 13], chromosomal abnormalities, unexplained mental retardation, multiple miscarriages, and major birth defects. Any increase above the baseline population risk would depend upon the precise condition in the family, the inheritance pattern, and the degree of relatedness to the woman or her partner. For familial conditions that are the result of mutations in single genes or inheritance of unbalanced chromosomal constitutions from carriers of balanced chromosomal rearrangements, preconception care should include offering genetic testing to determine if the woman and/or her partner is a carrier for the condition, which might substantially increase the genetic risk for a future pregnancy. Numerous advantages derive from discussing these risks and performing appropriate testing in the preconception period rather than during a pregnancy. These include 1) having adequate time available to obtain test results from those with long turnaround times and to obtain follow-up testing, if required, 2) not being limited by gestational age for the performance of specific prenatal diagnostic options, and 3) allowing discussions of reproductive planning to occur without the concern about the outcome of an ongoing pregnancy.

An ethnic background assessment is also part of a detailed family history, since certain genetic conditions are more prevalent in individuals of particular backgrounds. For example, individuals of Ashkenazi Jewish ancestry should be informed of their possible increased risk to have an infant with conditions such as Tay-Sachs disease, cystic fibrosis, Gaucher disease, and several other conditions [14]. Caucasians, particularly those of northern European ancestry, should be informed about a possible increased risk of cystic fibrosis [15]. African-Americans should be informed about the risk of sickle cell disease [16]. Finally, individuals of Mediterranean ancestry should be informed about the risk of thalassemia in an infant [16]. For a woman who is at higher risk for having children with these and/or other conditions, genetic testing is appropriate to determine if she is a carrier for the condition of interest. Knowing that she is a carrier, which increases her risk of having a child with the condition, allows for more informed reproductive planning. Often, when a woman is found to be a carrier, her partner also requires testing to provide the couple with the most accurate risk assessment for having an affected offspring. In contrast, if genetic testing finds that the woman is not a carrier, her risk is substantially decreased, which would improve the chance for a normal outcome in a future pregnancy.

Another factor that commonly increases the baseline population risk is maternal age. Women who are 35 years or older at the time of delivery (i.e., advanced maternal age) are considered to have an elevated risk of having an infant with a chromosomal abnormality, such as Down syndrome. Age 35 years at the time of delivery is designated as advanced maternal age because at that age the risk of chromosome abnormalities exceeds the risk of a complication from the amniocentesis procedure used to diagnose the abnormality. Therefore, an amniocentesis or chorionic villus sampling procedure is routinely offered to women of advanced maternal age for prenatal diagnosis of chromosome abnormalities [17]. As with women who have risks associated with family history and ethnic background, women with advanced maternal age should receive, as part of their standard preconception care, information about the genetic risks to a pregnancy and the available prenatal diagnostic options. A significantly increased risk for chromosome abnormalities is not associated with advance paternal age. However, as a man reaches the age of approximately 40 years, his risk of having an infant with one of several autosomal dominant conditions (achondroplasia, Apert syndrome, Marfan syndrome, and others reviewed in [18] and [19]), increases above the baseline population risk [20]. This risk information should also be incorporated into standard preconception genetic counseling and risk assessments, although the advanced paternal age risk is quite small compared to the significant risk for chromosome abnormalities that occurs with advanced maternal age.

The second major category of preconception genetic issues is the provision of appropriate preconception care and counseling for women with known or suspected genetic disorders in order to decrease the morbidity and mortality for the women during a future pregnancy, and to lessen the risk for avoidable complications in the infant. As healthcare has improved for infants and children with genetic disorders, many reach adulthood and women with a variety of genetic disorders are able to conceive and carry a pregnancy. However, because of their genetic condition, such women are at increased risk for maternal complications during a pregnancy. Examples include the development of pulmonary insufficiency in a woman with cystic fibrosis, sickle cell crisis and other vascular complications in a woman with sickle cell disease, 
hypoglycemic seizures in women with fatty acid oxidation disorders or glycogen storage disease type Ia, and hyperammonemia and encephalopathy in women with ornithine transcarbamylase deficiency. For each of these and many other genetic conditions, inadequate prenatal and postpartum care and monitoring could lead to significant morbidity or even death. Without doubt, women with genetic conditions need to be aware of the added health risk during a future pregnancy, and they should be monitored by appropriate obstetrics or maternal-fetal medicine specialists.

Women with certain genetic metabolic conditions have an increased risk of congenital anomalies and/or medical problems in their infants, particularly if their metabolic disorder is not appropriately managed during a pregnancy or if they do not follow the prescribed dietary and medication regimen. A well- described example is maternal phenylketonuria (PKU) embryopathy. Pregnant women with PKU or milder elevations of blood phenylalanine (hyperphenylalaninemia) who do not follow a low-phenylalanine diet and take the medical formula supplement will have high blood levels of phenylalanine. Elevated phenylalanine levels are teratogenic and can cause infants to have microcephaly, mental retardation, behavioral problems, and congenital heart defects [21, 22]. Women with such genetic metabolic conditions should be counseled and educated accordingly during preconception care, and plans should be developed so that women with these conditions can be in optimal metabolic control even before becoming pregnant.

\section{Summary}

Genetic issues are too important to be ignored during preconception care since they can have quite profound implications for a woman's future pregnancies. An important recommended intervention is a complete family history assessment, either though a self-assessment tool that is reviewed by the physician, or through a questionnaire administered by a healthcare provider. The utility and success of family history tools in assessing risk are well established in other medical areas $[23,24]$ and should be similarly effective in the genetic assessment of risk as part of preconception care. Genetic counseling information should be provided regarding the baseline population genetic risk to a pregnancy, as well as additional risks revealed by family history, ethnic background, and, if applicable, advanced maternal and/or paternal ages. Appropriate carrier testing should be offered, with the understanding that a negative carrier test result, in the case of some DNA-based tests, does not exclude the possibility that the individual tested is a carrier. Women with an increased risk of having an infant with a birth defect or genetic condition should be referred to a clinical genetics provider for further counseling and information about pregnancy options. Finally, women who themselves have a genetic condition should receive appropriate preconception and prenatal care, education, and medical counseling to avoid morbidity and mortality for themselves and their future pregnancies.

\section{References}

1. American College of Obstetricians and Gynecologists. The importance of preconception care in the continuum of women's health care. ACOG committee opinion 313. Washington, DC: ACOG; 2005.

2. Czeizel AE. Ten years of experience in the periconceptional care. Eur J Obstet Gynec Reprod Biol 1999;89:43-9.

3. Czeizel AE, Gasztonyi Z, Kuliev A. Periconceptional clinics: a medical health care infrastructure of new genetics. Fetal Diagn Ther 2005;20:515-8.

4. Myrianthopoulos NC, Chung CS. Congenital malformations in singletons: epidemiologic survey. Report from the Collaborative Perinatal project. Birth Defects Orig Artic Ser 1974;10:1-58.

5. Christianson RE, van den Berg BJ, Milkovich L, Oechsli FW. Incidence of congenital anomalies among white and black live births with long term follow-up. Am J Pub Health 1981;71:133341.

6. Van Regemorter N, Dodion J, Druart C, Hayez F, Vamos E, Flament-Durand J, Perlmutter-Cremer N, Rodesh F. Congenital malformations in 10,000 consecutive births in a university hospital: need for genetic counseling and prenatal diagnosis. J Pediatr 1984;104:386-90.

7. Balkite EA, Puck SM. Prenatal genetic risk assessment: A guide for health professionals. Cambridge, MA: Genzyme Genetics, Genzyme Corporation; 2003.

8. de Weerd S, van der Bij AK, Cikot RJLM, Braspenning JCC, Braat DDM, Steegers EAP. Preconception care: a screening tool for health assessment and risk detection. Prev Med 2002;34:50511.

9. Program ME. Genetic risk screening office guide. Scarborough, ME: Foundation for Blood Research; 1998.

10. Prenatal Screening Questionnaire. American Medical Association: Family History Tools, 2006. (http://www.amaassn.org/ama/pub/category/13332.html).

11. Family Health and Social History. March of Dimes: Genetics and Your Practice, 2006. (http://www.marchofdimes.com/ gyponline/index.bm2).

12. American College of Medical Genetics. Fragile X syndrome: Diagnostic and carrier testing. Working group of the genetic screening subcommittee of the clinical practice committee. Bethesda, MD: ACMG; 1994.

13. American College of Obstetricians and Gynecologists. Fragile $\mathrm{X}$ syndrome. ACOG committee opinion 161. Washington, DC: ACOG; 1995.

14. American College of Obstetricians and Gynecologists. Prenatal and preconceptional carrier screening for genetic diseases in individuals of eastern European Jewish descent. ACOG committee opinion 298. Washington, DC: ACOG; 2004.

15. Cystic Fibrosis Genetic Analysis Consortium. Population variation of common cystic fibrosis mutations. Hum Mutat 1994;4:167-77.

16. American College of Obstetricians and Gynecologists. Genetic screening for hemoglobinopathies. ACOG committee opinion 238. Washington, DC: ACOG; 2000.

17. American College of Obstetricians and Gynecologists. Prenatal diagnosis of fetal chromosomal abnormalities. ACOG practice bulletin 27. Washington, DC: ACOG; 2001.

18. Crow JF. The origins, patterns and implications of human spontaneous mutation. Nat Rev Genet 2000;1:40-7. 
19. Rolf C, Nieschlag E. Reproductive functions, fertility and genetic risks of ageing men. Exp Clin Endocrinol Diabetes 2001;109:6874.

20. American College of Medical Genetics. Statement on guidance for genetic counseling in advanced paternal age. ACMG Newsletter 1996;6:13.

21. Lenke RR, Levy HL. Maternal phenylketonuria and hyperphenylalaninemia: an international survey of the outcome of untreated and treated pregnancies. N Engl J Med 1980;303:1202-8.
22. Drogari E, Smith I, Beasley M, Lloyd JK. Timing of strict diet in relation to fetal damage in maternal phenylketonuria. An international collaborative study by the MRC/DHSS Phenylketonuria Register. Lancet 1987;2:927-30.

23. Johnson J, Giles RT, Larsen L, Ware J, Adams T, Hunt SC. Utah's family high risk program: bridging the gap between genomics and public health. Prev Chronic Dis 2005;2:A24-30.

24. Tyagi A, Morris J. Using decision analytic methods to assess the utility of family history tools. Am J Prev Med 2003;24:199-207. 